\title{
Ensino de Lógica e Programação no Ensino Médio por meio de uma Abordagem Lúdica e Gamificada
}

\author{
Maurício M. Arimoto ${ }^{1}$, José Henrique R. Cruz ${ }^{1}$ \\ ${ }^{1}$ Centro de Ciências Tecnológicas - Universidade Estadual do Norte do Paraná (UENP) \\ Caixa Postal 261 - 86360-000 - Bandeirantes - PR - Brasil \\ \{mauricioarimoto, henriquericordi\}egmail.com
}

\begin{abstract}
This paper presents an experience report on the application of a playful and gamified approach, focused on the introduction of logic and programming computers in a public school. The application took place through programming workshops, carried out during shiflafter school and involving the participation of 32 students from high school. The results indicate that the use of such approach tends to make learning more attractive, and encourages student's motivation and engagement during the knowledge acquisition process.
\end{abstract}

Resumo. Nesse trabalho é apresentado um relato de experiência da aplicação de uma abordagem lúdica e gamificada, voltada para a introdução de lógica e programação de computadores em uma escola da rede pública de ensino. A aplicação ocorreu por meio oficinas de programação, realizadas no contraturno do período de ensino regular e envolvendo a participação de 32 alunos do ensino médio. Os resultados obtidos indicam que o uso de tal abordagem tende a tornar a aprendizagem mais atrativa, além de fomentar a motivação e o engajamento dos alunos durante o processo de aquisição de conhecimento.

\section{Introdução}

A lógica e a programação de computadores fazem parte da vida de todos, direta ou indiretamente. A primeira está presente em todas as atividades diárias, enquanto a segunda é fundamental no desenvolvimento, manutenção e melhoria das tecnologias. Dessa forma, elas são vista como habilidades fundamentais, não servindo apenas para preparar mão de obra para a área da tecnologia [Sousa 2013].

Discussões relacionadas ao ensino de lógica e programação vão além da educação em nível superior. Um tema recorrente de debate é a introdução da disciplina de programação no currículo escolar, sobretudo no ensino fundamental e médio. Almeja-se, com isso, tanto o crescimento pessoal e social do cidadão como a qualidade de sua formação.

Entretanto, a aprendizagem de programação não pode ser considerada trivial, pois os conceitos abordados e a carga de conhecimentos agregados não são, em sua maioria, simples e de fácil compreensão, especialmente para alunos da educação básica [Arimoto and Oliveira 2019, Santiago and Kronbauer 2016]. Neste contexto, abordagens baseada em gamificação trazem uma nova perspectiva para o processo de ensino e aprendizagem, dado que jogos digitais ou elementos típicos de jogos são integrados ao processo educacional (princípio da gamificação) [Fotaris et al. 2016], tornando a experiência de aprendizagem do aluno mais atrativa.

Estudos conduzidos na literatura ressaltam aspectos positivos da gamificação no contexto educacional, incluindo: (1) aumento da motivação dos estudantes; (2) 
desenvolvimento cognitivo e intelectual dinâmico (sobretudo de crianças e adolescentes); (3) desenvolvimento do pensamento algorítmico; e (4) elevação da habilidade para resolver problemas [Dantas 2013].

Neste trabalho, apresenta-se uma experiência na aplicação de oficinas para a introdução de lógica e programação de computadores na educação básica por meio de uma abordagem lúdica e gamificada. O trabalho está organizado da seguinte forma: na Seção 2 são sintetizados os trabalhos relacionados; na Seção 3 são descritos o planejamento e execução das oficinas; na Seção 4 apresenta-se a análise e síntese dos resultados alcançados; já na Seção 5 apresentam-se as considerações finais sobre o trabalho realizado.

\section{Trabalhos Relacionados}

[Brum and Cruz 2017] relataram uma experiência no uso de gamificação como metodologia na disciplina facultativa de robótica, sendo utilizados os kits de LEGO Mindstorms. O projeto contou com a participação de 21 alunos do ensino fundamental. De acordo com os autores, os resultados demonstraram melhoria significativa no aprendizado do conteúdo abordado na disciplina de robótica, promovendo o engajamento e a cooperação entre os alunos. Seguindo essa linha, [Farias et al. 2018] apresentaram uma proposta de gamificação da disciplina lógica de programação e sua respectiva aplicação em uma turma de um curso técnico de informática. Os autores afirmaram que houve um maior engajamento e empenho dos alunos na disciplina, e ao final, houve um aumento do percentual de aprovados na disciplina.

[Silva et al. 2017] apresentaram um relato de experiência no ensino de programação na educação básica por meio do uso de storytelling juntamente com a gamificação, além da linguagem Python integrada ao jogo Minecraft. Partiparam do projeto, sete alunos do ensino fundamental e médio. De acordo com os autores, os resultados mostraram que a estratégia adotada para o ensino de programação foi avaliada positivamente pelos alunos. Outro trabalho similar é apresentado em [Silva et al. 2018], em que a gamificação é utilizada com storytelling e a linguagem de programação C. Os autores concluiram que é possível desenvolver o aprendizado de programação utilizando-se de elementos de jogos para estimular determinadas habilidades cognitivas nos alunos do século XXI.

Apesar da aplicação de técnicas e elementos característicos de jogos no âmbito educacional ter ganhado crescente importância, elas ainda se mostram bastante incipientes, especialmente no contexto de introdução da programação de computadores no ensino médio.

\section{Planejamento e Execução das Oficinas}

Uma reunião foi marcada com a diretoria e equipe pedagógica de uma escola da rede pública de ensino para discussão sobre a proposta das oficinas e a definição das datas e horários para a sua realização. A proposta foi apresentada junto aos alunos do ensino médio $\left(1^{\mathrm{a}}, 2^{\mathrm{a}}\right.$ e $3^{\mathrm{a}}$ séries) visando a seleção de participantes. Essa etapa foi importante para despertar o interesse dos alunos pelas oficinas de programação. Ao final, foram selecionados 30 alunos com idades entre 15 e 20 anos. As oficinas foram realizadas no contraturno do período de ensino regular, compreendendo 30 horas divididas em quatro semanas no período de um mês.

Um questionário de diagnóstico foi aplicado para verificar o grau de conhecimento dos alunos sobre os temas relacionados ao projeto e identificar as potenciais dificuldades. Os resultados mostraram que boa parte dos alunos havia tido pouco contato com programação, além de pouca informação sobre a gamificação e seu uso na aprendizagem de programação. 
Para as oficinas de programação, inicialmente foram levantados elementos de gamificação aplicáveis ao ensino e aprendizagem de programação. Durante esse levantamento, materiais de apoio foram criados em diferentes tipos de mídias, como: animações, textos expositivos da ferramenta utilizada (linguagem de programação $\mathrm{C}++$ ), demonstração de exemplos em laboratório e construção dos algoritmos em conjunto com os estudantes (utilizando-se de exemplos do mundo real) e, por fim, exercícios para a prática e nivelamento de aprendizagem dos estudantes.

Foram abordados conceitos introdutórios de programação na linguagem $\mathrm{C} / \mathrm{C}++$ (Figura 11) utilizando-se de elementos de gamificação como: rankings, atividades lúdicas, possibilidade de medição de desempenho individual, entre outros, em que se procurou reforçar as ideias de recompensas e estimular a competitividade e a motivação no processo de aprendizagem.
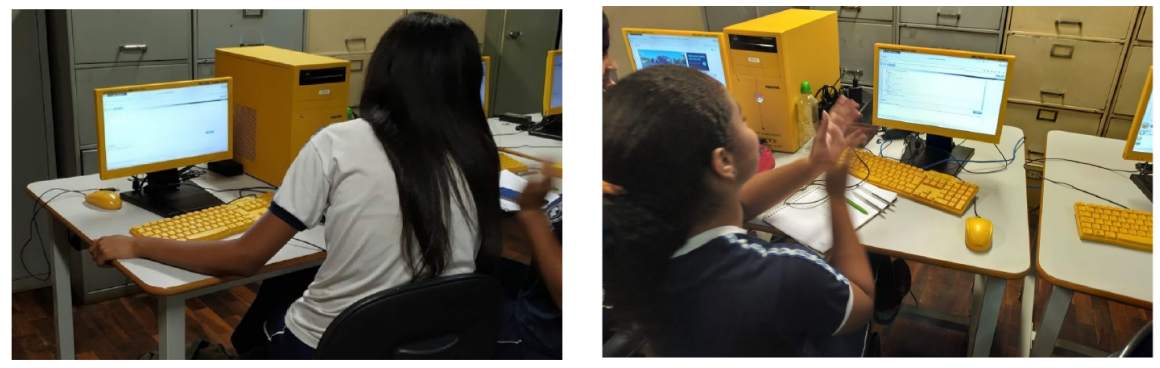

Figura 1. Oficina de programação com abordagem lúdica e gamificada

Considerando os recursos apresentados, foi construída uma plataforma web utilizando-se da abordagem gamificada para a apresentação desses recursos (Figura 2). Além disso, foi realizada a verificação de aprendizagem dos estudantes, de modo que as diferentes atividades aplicadas por meio de questões teóricas e construção de algoritmos foram estruturadas de forma a gerar rankings/placares, aos quais resultaram em recompensas (princípio da gamificação).

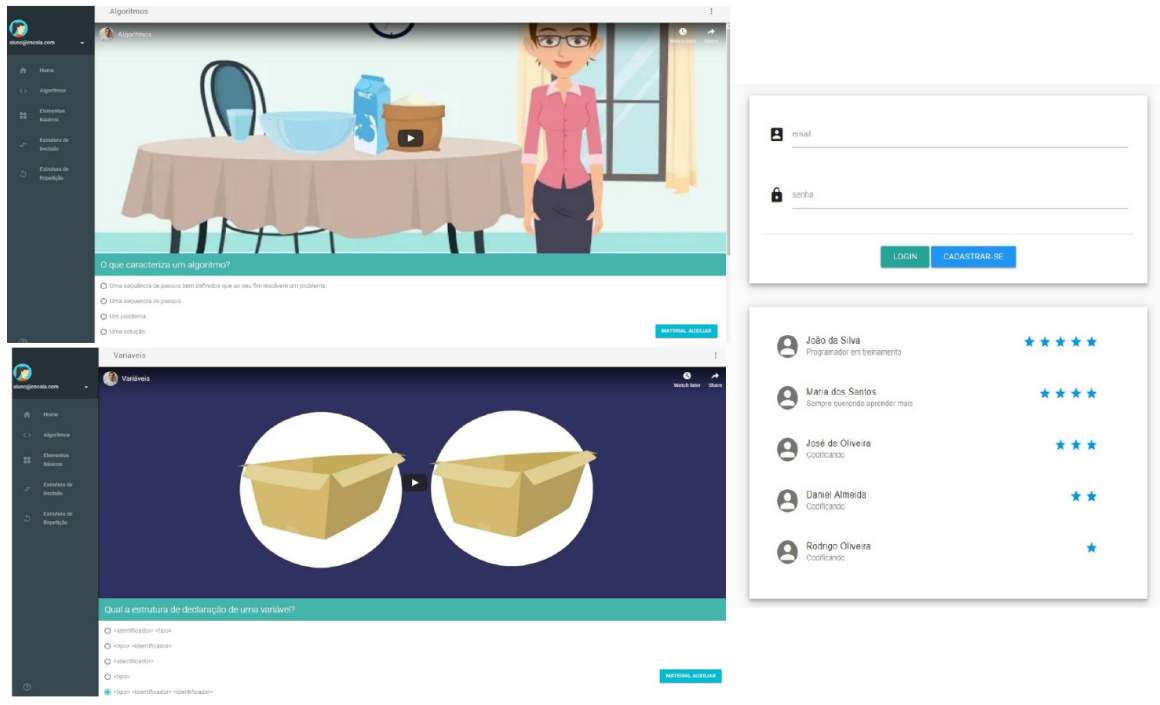

Figura 2. Telas da plataforma de aprendizagem

O cronograma de execução da oficina é apresentado na Tabela 1. No primeiro tópico foram introduzidos conceitos básicos da gamificação e como ela poderia ser aplicada ao ensino de programação. No segundo tópico foram abordados conceitos básicos de algoritmos. Também foram introduzidos os componentes básicos da linguagem $\mathrm{C}++$, 
incluindo a estrutura de programas, variáveis e tipos e comandos de entrada e saída. Além disso, foi introduzida a plataforma web construída para auxiliar no ensino de programação. No terceiro tópico foram abordadas as estruturas de decisão através dos comandos de decisão. Por fim, foram abordadas as estruturas de repetição através dos comandos de repetição.

Tabela 1. Cronograma de execução das oficinas

\begin{tabular}{|c|c|c|c|c|}
\hline \multirow{2}{*}{ Tópicos } & \multicolumn{4}{|c|}{ Carga horária } \\
\cline { 2 - 5 } & $1^{\mathrm{a}}$ semana & $2^{\mathrm{a}}$ semana & $3^{\mathrm{a}}$ semana & $4^{\mathrm{a}}$ semana \\
\hline 1. Noções básicas de gamificação & $2 \mathrm{~h}$ & & & \\
\hline 2. Componentes básicos do programa & $6 \mathrm{~h}$ & $2 \mathrm{~h}$ & & \\
\hline 3. Comandos de decisão & & $6 \mathrm{~h}$ & $4 \mathrm{~h}$ & \\
\hline 4. Comandos de repetição & & & $4 \mathrm{~h}$ & $6 \mathrm{~h}$ \\
\hline
\end{tabular}

\section{Resultados e Discussões}

A apresentação dos resultados baseia-se nas respostas de todos os participantes, incluindo os alunos das $1^{\mathrm{a}}, 2^{\mathrm{a}}$ e $3^{\mathrm{a}}$ séries do segundo grau. Analisando o desempenho dos alunos, pode-se observar que cerca de $84,4 \%$ dos participantes conseguiram compreender bem e aplicar na prática os conceitos apresentados, enquanto que 15,6\% compreenderam de maneira satisfatória. Dentre as principais dificuldades enfrentadas pelos alunos, destaca-se o entendimento de conceitos de lógica de programação.

Os alunos foram questionados se questões como pontos, rankings e recompensas foram úteis para aumentar a motivação em aprender programação. Observou-se que 26 alunos (81,2\%) (Figura 3a) afirmaram que a aplicação de tais elementos foram fatores motivadores para a aprendizagem. Além disso, 25 alunos $(78,1 \%)$ (Figura 3b) consideraram como sendo relevante a utilização de uma abordagem lúdica e gamificada na aprendizagem de programação.

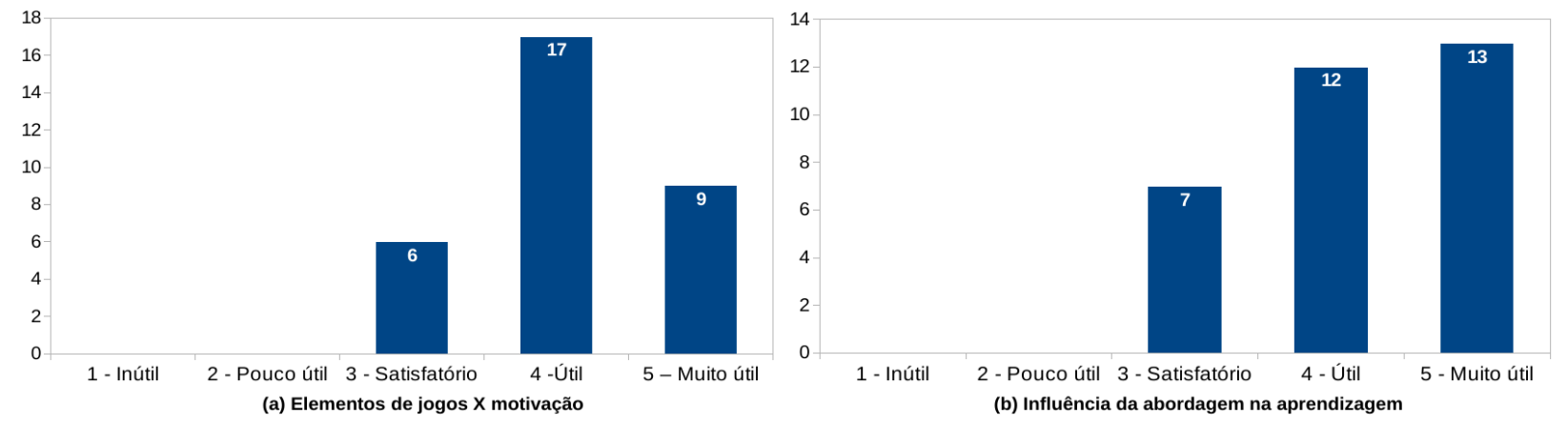

Figura 3. Influência da gamificação na aprendizagem

Antes das execução das oficinas os alunos haviam demonstrado pouco interesse por programação por considerarem a sua aprendizagem complexa e por apresentrarem uma visão equivocada sobre a mesma. Após a aplicação das oficinas, cerca de $65 \%$ dos alunos demonstrou maior interesse na área de programação. Nesse sentido, os alunos também foram questionados sobre a possibilidade de ingressarem em algum curso da área, sendo que aproximadamente $43 \%$ deles consideraram tal possibilidade.

De modo geral, observou-se que a abordagem favoreceu o engajamento dos alunos, além de fornecer uma visão mais próxima da realidade do que se trata a programação de computadores, sendo que muitos alunos possuíam uma visão equivocada sobre o assunto, muitas vezes ocasionada por algum estereótipo presente na cultura. 


\section{Conclusões}

Neste trabalho, foi apresentado um relato de experiência da introdução de conceitos de lógica e programação por meio de uma abordagem lúdica e gamificada. Para isso, oficinas de programação foram conduzidas em uma escola da rede pública de ensino. O público-alvo envolveu 32 alunos das $1^{\mathrm{a}}, 2^{\mathrm{a}}$ e $3^{\mathrm{a}}$ séries do ensino médio.

A abordagem utilizada influenciou positivamente na aprendizagem dos alunos, aumentado a motivação e o engajamento durante as aulas e despertando um maior interesse por programação. A experiência apresentada mostrou-se interessante por apresentar resultados positivos, sem grande esforço de adaptação no processo de ensino de aprendizagem, apesar de não ser amplamente aceita no contexto da educação básica.

Alguns alunos tiveram dificuldades em compreender conceitos inerentes à lógica e programação devido à sua complexidade. Nesse sentido, o pouco tempo dedicado às oficinas pode ter sido um empecilho. Assim, novas oficinas de programação serão executadas, considerando uma carga maior de aulas.

\section{Agradecimentos}

À Fundação Araucária pelo apoio financeiro - Programa de Apoio à Inclusão Social (PIBIS).

\section{Referências}

Arimoto, M. M. and Oliveira, W. T. (2019). Dificuldades no processo de aprendizagem de programação de computadores: um survey com estudantes de cursos da área de computação. pages 244-254. 27 Workshop sobre Educação em Computação - Congresso da Sociedade Brasileira de Computação.

Brum, M. G. and Cruz, M. K. (2017). Gamificação para o ensino de computação na educação básica. In $26^{\circ}$ Workshop sobre Educação em Computação (WEI), XXXVII Congresso Brasileiro da Sociedade Brasileira de Computação (CBSC), pages 2100-2109.

Dantas, V. F. e. a. (2013). Combinando desafios e aventura em um jogo para apoiar a aprendizagem de programação em vários níveis cognitivos. In Anais do Simpósio Brasileiro de Informática na Educação, volume 24.

Farias, C. M., Azevedo, F. P., and dias, J. E. J. (2018). Uma abordagem gamificada para o ensino de lógica de programação: relato de experiência. In $27^{\circ}$ Workshop sobre Educação em Computação (WEI), XXXVIII Congresso Brasileiro da Sociedade Brasileira de Computação (CBSC), pages 1-10.

Fotaris, P., Mastoras, T., Leinfellner, R., and Rosually, Y. (2016). Climbing up the leaderboard: an empirical study of applying gamification techniques to a Computer Programming Class. The Electronic Journal of e-Learning, 14(2):94-110.

Santiago, A. D. V. and Kronbauer, A. H. (2016). Um modelo lúdico para o ensino de conceitos de programação de computadores. In Simpósio Brasileiro de Informática na Educação (SBIE), pages 420-42.

Silva, J. A. L., Oliveira, F. C. S., and Martins, D. J. S. (2017). Storytelling e gamificação como estratégia de motivação no ensino de programação com python e minecraft. In Proceedings of SBGames 2017, pages 987-990.

Silva, J. A. L., Oliveira, F. C. S., and Martins, D. J. S. (2018). Gamificação e storytelling como estratégia de motivação no ensino de programação. In Proceedings of SBGames 2018, pages 1097-1106. 